and who exhibit stereotyped movements, speech and language disorders, tics, and motor clumsiness. Asperger's disorder may overlap or occur concurrently with Tourette syndrome, pervasive developmental disorder, and attentional deficit disorders.

\title{
DEVELOPMENTAL DYSCALCULIA
}

An 11-year-old, Hebrew speaking boy of normal intelligence, referred to Alyn Hospital, and Schneider Children's Medical Center, Jerusalem, for evaluation of learning and attentional problems, was found to have a profound dyscalculia based on a proposed lack of "cardinal/ordinal skills acquisition device" (COSAD). Several male family members had dysgraphia, right-left disorientation, and dyslexia. At birth, the child was hypotonic, and motor development was delayed, walking independently at 2 and $1 / 2$ years. Neurologic abnormalities included high-pitched voice, dysgraphia, right-left disorientation, finger agnosia, clumsiness in running and jumping, scoliosis, and fine motor incoordination. At 4 years, he developed grand mal seizures treated with carbamazepine, and at 7 years he received pemoline (Cylert) for ADD without hyperactivity. The pemoline benefited overall functioning. The use of linguistic, visual and verbal memory cues compensated for deficits in ordinal number use, but not for cardinal number skills, which remained limited. He could count small numbers, but could not do simple calculations, a skill requiring an innate experience of quantity, less amenable to language, visual-spatial, or logical mediation. Different theories of number development and mathematical skills are discussed in relation to mechanisms of dyscalculia and its management. (Ta'ir J, Brezner A, Ariel R. Profound developmental dyscalculia: evidence for a cardinal/ordinal skills acquisition device. Brain and Cognition November 1997;35:184-206). (Reprints: Judy Ta'ir, Alyn Hospital, PO Box 9117, Jerusalem 91090, Israel).

COMMENT. A child with ADHD and learning disability involving mathematical skills should be examined neurologically for Gerstmann's syndrome (dyscalculia, dysgraphia, right-left disorientation, and finger agnosia). In adults with stroke or cerebral tumor, GS is usually associated with lesions localized to the angular gyrus and parietal lobe of the dominant hemisphere. In children, partial Gerstmann syndromes may be developmental or acquired and may occur with focal lesions in other areas of the dominant hemisphere or with diffuse involvement of either or both hemispheres. An EEG may help in localizing a lesion in children with ADHD, GS, and seizures, and may prompt the need for an MRI.

Profound developmental dyscalculia may occur in children of normal or even superior IQ Deficits in specific cognitive areas may involve visualspatial perception and parietal-occipital dysfunction. In a theoretical hypothesis of development dyscalculia, an innate, highly specific cognitive domain is involved; ordinal number tasks and counting small magnitudes may be successfully completed, whereas larger quantities involving calculations are not possible.

\section{CHILDHOOD ADHD AND ADULT CRIMINALITY}

The relationship between attention deficit hyperactivity disorder in childhood and criminality in adolescence and adulthood was examined in 89 hyperactive and 87 normal control subjects studied prospectively at the Division of Child Psychiatry, Oregon Health Sciences University, Portland, OR. Hyperactive subjects had significantly higher juvenile and adult rates of 